\title{
Differentiation between temporary and real non-clearability of biotinylated IgG antibody by avidin in mice
}

\section{Shuping Dou ${ }^{1}$, John Virostko ${ }^{2}$, Mary Rusckowski ${ }^{1}$, Dale L. Greiner ${ }^{3}$, Alvin C. Powers ${ }^{4,5,6}$ and Guozheng Liu ${ }^{1 *}$}

${ }^{1}$ Department of Radiology, University of Massachusetts Medical School, Worcester, MA, USA

2 Vanderbilt University Institute of Imaging Science, Vanderbilt University, Nashville, TN, USA

${ }^{3}$ Department of Molecular Medicine, University of Massachusetts Medical School, Worcester, MA, USA

${ }^{4}$ Division of Diabetes, Endocrinology, and Metabolism, Department of Medicine, Vanderbilt University, Nashville, TN, USA

${ }^{5}$ Department of Molecular Physiology and Biophysics, Vanderbilt University, Nashville, TN, USA

${ }^{6}$ Veterans Affairs Tennessee Valley Healthcare System, Nashville, TN, USA

\section{Edited by:}

Chiranjib Chakraborty, Galgotias

University, India

Reviewed by:

Rahman M. Mizanur, US Army Medical Research Institute of

Infectious Diseases, USA

Miaozong Wu, Marshall University, USA

\section{${ }^{*}$ Correspondence:}

Guozheng Liu, Department of

Radiology, University of

Massachusetts Medical School,

55 Lake Avenue North, Worcester,

MA 01655-0243, USA

e-mail:guozheng.liu@

umassmed.edu
Although an increasing number of antibody conjugates are being used in the clinic, there remain many unmet needs in antibody targeting. Normal tissue background is one of the key issues that limits the therapeutic efficacy and the detection sensitivity. Background reduction coupled with dose increase may provide the required target accumulation of the label or toxin at an acceptable normal tissue background. However, the knowledge about the in vivo interaction between antibody and a clearing agent is currently inadequate for designing a rational clearance regimen or system. The current investigation focuses on the clearability of antibody for background reduction, an important topic to antibody targeting in general. The investigation employs pretargeting as a research tool and avidin as a model clearing agent. By comparing the effects of natural clearance at a longer post-injection time and avidin clearance, we demonstrated that avidin clearance is much more effective. By directly attaching avidin to a biotinylated antibody prior to injection, we found that the biotinylated antibody in blood, once bound to the clearing agent, can be removed from the circulation immediately and completely, while the real non-clearable antibody without biotin stays. The study of multiple avidin injections confirmed that the presence of clearable biotinylated antibodies after an avidin injection is due to their temporary inaccessibility and subsequent return from tissue compartments. The collective clearance efficiency of $91 \%$ by three avidin injections indicates a continuous IV infusion would be recommended to remove all of the biotinylated IgG molecules. In conclusion, the use of antibody pretargeting as a tool in this study has improved understanding of the incomplete clearance by avidin and can aid in overcoming this obstacle.

Keywords: pretargeting, antibody, clearing agent, avidin, in vivo accessibility, immunotargeting

\section{INTRODUCTION}

For targeted immunotherapy and immunodiagnosis, the clearance of normal tissue background is an important measure complementary to the enhancement of target accumulation. Although targeted immunochemotherapy of hematological cancer has achieved great success (Senter and Sievers, 2012; Deng et al., 2013), the relative poorer accessibility of antibody to solid tumors remains a challenge. Reducing the normal tissue background may allow for increasing the dose of the "warhead" or the target toxicity and therefore may improve solid tumor treatment. The background reduction is also critical for imaging the islets of Langerhans (Liu et al., 2011, 2012). Because islets constitute only $1-2 \%$ of the pancreas mass and the current nuclear imaging technologies cannot differentiate islets from non-islet pancreatic tissues, reduction of the non-specific binding in the exocrine tissues is crucial to assure the pancreas signal reflects the beta cell accumulation.
Currently, there are two clearing mechanisms in the literature used for reducing the normal tissue background. One mechanism using a secondary-antibody takes advantage of the large size of the aggregate formed with the pretargeting antibody. The aggregate can be removed from the circulation by reticuloendothelial (RE) cells (Goodwin et al., 1994, 1988). The other mechanism employs a clearing agent bearing galactosyl groups. Such clearing agents can be avidin (Yao et al., 1995; Mirallie et al., 2005; Liu et al., 2010), galactosylated antiantibodies against the pretargeting antibody (Sharkey et al., 1997), or galactosylated and biotinylated HSA (Axworthy et al., 2000). The complex formed between the antibody and clearing agent can be removed by an asialoglycoprotein receptor specific for the galactosyl groups (Ashwell and Morell, 1974; Ong et al., 1991). Both mechanisms traffic the circulating pretargeting molecules into liver. Most studies in the literature focus on the development of technologies that include a clearance step 
(Ashwell and Morell, 1974; Goodwin et al., 1988, 1994; Ong et al., 1991; Yao et al., 1995; Karacay et al., 1997; Sharkey et al., 1997; Axworthy et al., 2000; Wang et al., 2001; Mirallie et al., 2005; Liu et al., 2010). However, few efforts have been made to understand the in vivo interaction between the antibody and clearing agent (Kobayashi et al., 1995; Yao et al., 1995; Sharkey et al., 1997).

The clearance concept has been used for many years, but the current knowledge remains inadequate for readily designing a targeting system with a clearance step to achieve low blood background. The current investigation focuses on the clearability of biotinylated antibody using avidin as a clearing agent. It is known that avidin does not clear biotinylated antibody completely, but there is no quantitative study as to the exact cause. However, this topic is very important not only for developing a pretargeting technology with clearance, but also for any antibody-based drug for which the background is a concern.

In the current investigation, we employed a model pretargeting system to investigate the in vivo chemistry between avidin and biotinylated IgG antibody. The aim of this study is not to develop an improved pretargeting protocol but to understand the clearability of biotinylated antibody by avidin. As such, the pretargeting system is used as a research tool for understanding rather than solely for improved pretargeting technology. In this pretargeting system, the antibody CC49 is conjugated concomitantly with a biotin and a morpholino phosphorodiamidate oligomer (MORF). The biotin functions to bind to the clearing agent of avidin, whereas the MORF is for radiolabeling both in vivo and in vitro. In contrast to most clearance systems, the current design has avoided the binding competition between the clearing agent and the effector. In this investigation, normal mice are used to focus on the determinants of antibody clearance. Tumor accumulation will not be compromised by antibody clearance as previously concluded (Liu et al., 2010).

\section{MATERIALS AND METHODS}

The MORF and CMORF (complementary MORF) were customsynthesized by Gene Tools, LLC (Philomath, OR). Their base sequences and amine derivatization were the same as previously reported (Liu et al., 2010). The $\mathrm{EZ}^{\mathrm{TM}}$ Biotin Quantitation Kit and avidin were from Pierce (Thermo Fisher Scientific, Rockford, IL). The model antiTAG-72 IgG antibody CC49 was prepared by Strategic Biosolutions (Ramona, CA) from its murine hybridoma cell line (a gift from Dr. Jeff Schlom, Center for Cancer Research, NCI, NIH). The biotin-CC49-MORF was synthesized following the reactions illustrated below (Scheme 1) and characterized as previously described (Liu et al., 2010).

NHS-biotin + Ab + NHS-SANH $\longrightarrow$ biotin-Ab-SANH $\stackrel{\text { SFB-MORF }}{\longrightarrow}$ biotin-Ab-MORF

NHS: N-hydroxyl succinimidyl;

SANH: Acetone-protected pyridinyl hydrazine;

SFB: Succinimidyl 4-formyl benzoate;

MORF: Morpholino phosphorodiamidate oligomer

Scheme 1 | The reactions of antibody conjugation with both biotin and MORF.
The ${ }^{99 \mathrm{~m}} \mathrm{Tc}$-cMORF was prepared following a documented procedure (Liu et al., 2006). The ${ }^{99} \mathrm{Mo}-{ }^{99} \mathrm{~m}$ Tc generator was from Perkin Elmer Life Science Inc. (Boston, MA). All other chemicals were reagent grade and used without purification.

The concentrations of MORF and CMORF were measured by UV spectrophotometry using the molar absorbance values provided by the vendor. Size exclusion (SE) HPLC was used for the analyses of the MORF, cMORF and antibody. The HPLC system was equipped with a superpose-12 10/30 GL column (GE Healthcare Bio-Sciences AB, Uppsala, Sweden; optimal separation range: $1 \times 10^{3}$ to $3 \times 10^{5} \mathrm{Da}$ ), a $\mathrm{UV}$ in-line detector, and a radioactivity in-line detector. A $0.10 \mathrm{M} \mathrm{pH} 7.2$ phosphate buffer was used as the eluant at a flow rate of $0.60 \mathrm{~mL} / \mathrm{min}$. Radioactivity recovery was routinely measured and was always greater than $90 \%$.

We designed three animal studies in the current investigation. The animal use was approved by the IACUC of the University of Massachusetts Medical School. We first examined the natural background-reducing effect of time (prolonging pretargeting interval) and the use of avidin, to show the strength of clearing agent and to confirm the pharmacokinetic homogeneity of the antibody molecules after modification. We then studied the clearability of the biotin-CC49-MORF. The antibody was radiolabeled and bound to avidin prior to injection into mice, to mimic the avidin binding of antibody in blood. Based on the results from the first two studies, we designed a multiple avidin injection study to confirm that the biotinylated antibody that survived an avidin clearance was still clearable.

\section{CLEARANCE EFFICIENCY BY AVIDIN WITH VARYING PRETARGETING INTERVAL}

Because a longer wait (pretargeting interval prolongation) also reduces the non-specific background of antibody, we examined the effect of pretargeting interval prolongation along with avidin clearance. The statistical significance for the blood level changes by pretargeting interval prolongation and avidin clearance was analyzed by Two-Way analysis of variance (ANOVA) at the significant level of 0.05 . A new quantity for the effect of avidin (clearance efficiency) is defined as the \% $\mathrm{ID} / \mathrm{g}$ reduction by avidin of blood radioactivity as compared to the $\% \mathrm{ID} / \mathrm{g}$ without avidin (100\% - the blood level ratio with/without avidin injection). It can be calculated from the measured blood activity levels. The standard deviation of the clearance efficiency can be estimated by the uncertainty propagation formula:

$$
S D=\sqrt{S D_{\text {without }}^{2}\left(\frac{1}{\text { Blood }_{\text {with }}}\right)^{2}+S D_{\text {with }}^{2}\left(\frac{\text { Blood }_{\text {without }}}{\text { Blood }_{\text {with }}^{2}}\right)^{2}} .
$$

To label the antibody in vivo, the cMORF was injected in a many-fold molar excess as compared to the MORF on the antibody. The blood level of the CMORF was therefore proportional to that of the antibody and was a measure of the antibody blood level, but it is lower in value than the antibody level because only a small portion of the cMORF was consumed and the rest of the cMORFs were excreted rapidly. The relationship is: $\% \mathrm{ID} / \mathrm{g}_{\text {antibody }}=\% \mathrm{ID} / \mathrm{g}_{\mathrm{cMORF}} /($ molar dose ratio of cMORF/antibody $\times$ MORF number per antibody). 


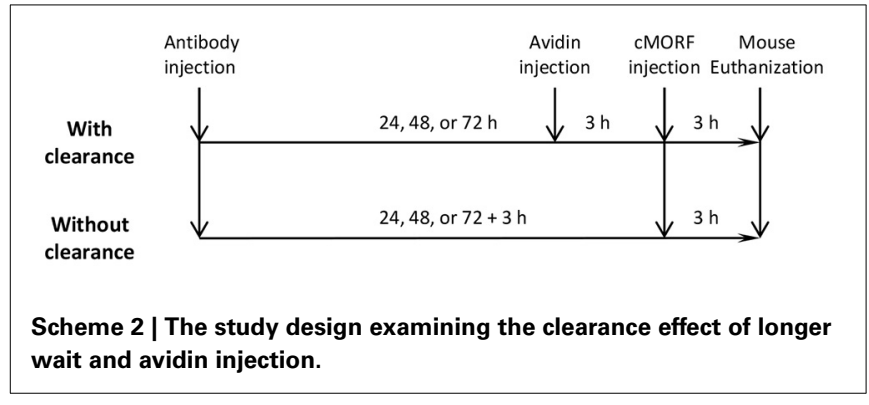

As shown in Scheme 2, in a study set of 3 groups of mice $(N=4)$, each of the 12 mice received via tail vein $30 \mu \mathrm{g}$ of biotinCC49-MORF (0.67 MORFs and 3.41 biotins per antibody); 1, 2 , and 3 days later, an avidin dose of $34 \mu \mathrm{g}$ was administrated intravenously to the 3 groups of 4 mice, followed $3 \mathrm{~h}$ later by the injection of $1.2 \mu \mathrm{g}(80 \mu \mathrm{Ci})$ of ${ }^{99} \mathrm{~m} \mathrm{Tc}$-cMORF. The mice were euthanized for biodistribution at $3 \mathrm{~h}$ subsequent to the radioactivity injection. In a control study set, an otherwise identical procedure was applied except for the removal of the avidin injection. The $1.2 \mu \mathrm{g}$ of cMORF was an optimized mass dose that was sufficient to saturate the MORF-antibody and the $34 \mu \mathrm{g}$ of avidin was previously determined to be more than sufficient to saturate the biotin-modified MORF-antibodies in the circulation (Liu et al., 2010). The decrease of blood radioactivity level of ${ }^{99} \mathrm{~m}$ TccMORF was used as the measure of background improvement as it was proportional to the decrease of the MORF-antibody level.

\section{AVIDIN CLEARABILITY OF THE BIOTIN-ANTIBODY-MORF}

As will be shown, although avidin clearance was much more effective as compared to the prolonged pretargeting interval, the clearance effect was still considerably low (50-60\%). Thus, we sought to determine whether a biotinylated antibody could be cleared completely once bound to avidin in blood. We designed a study to inject biotinylated antibody both radiolabeled and bound to avidin prior to the IV injection, to mimic the antibody in blood immediately after binding to avidin. Thus, a biotin-CC49MORF antibody (0.63 MORFs and 1.68 biotins per antibody) was radiolabeled by mixing with ${ }^{99 \mathrm{~m}} \mathrm{Tc}$-cMORF (at a molar ratio of MORF/cMORF = 3/1). The mixture was purified over a G100 size-exclusion column. The radiolabeled biotin-CC49-MORF was then allowed to combine with excess avidin (molar ratios of avidin/biotin $=5$ ). The pharmacokinetics of the both radiolabeled and avidin-bound antibody was then measured. Mice in 4 groups $(N=4$, each group) each received an IV injection of the mixture containing $12.5 \mu \mathrm{g}(9 \mu \mathrm{Ci})$ of ${ }^{99} \mathrm{~m}$ Tc-labeled biotin-CC49-MORF and $45 \mu \mathrm{g}$ of avidin. At $2 \mathrm{~min}, 30 \mathrm{~min}, 1.5 \mathrm{~h}$, and $2.5 \mathrm{~h}$, the mice were euthanized for measuring the biodistribution. In a control set also consisting of 4 groups ( $N=4$, each group), an identical procedure was performed except avidin was not added to the injectate.

\section{MULTIPLE INJECTIONS OF AVIDIN}

As will be shown, the clearance efficiency in the first study was less than the clearable portion measured in the second study. We thus sought to determine whether the non-cleared portion is

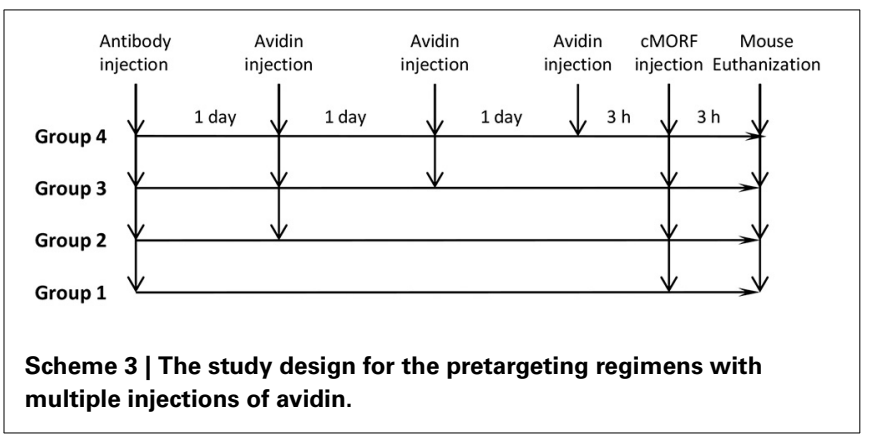

temporary non-clearable due to the sequestration of the antibody in the extravascular space. As shown in Scheme 3, each mouse of 4 groups $(N=4)$ was injected with $30 \mu \mathrm{g}$ of biotin-CC49-MORF (0.63 MORFs and 1.68 biotins per antibody). One day after antibody injection, mice in groups 2, 3, and 4 were injected with $34 \mu \mathrm{g}$ of avidin. Two days later, the mice in groups 3 and 4 received another avidin injection. Three days later, mice in group 4 were given the third avidin injection. At $3 \mathrm{~h}$ following the last avidin injection of group 4, all mice in groups 1-4 received the same dose of radiolabeled cMORF $(1.1 \mu \mathrm{g}, 40 \mu \mathrm{Ci})$ and all mice were euthanized for biodistribution at $3 \mathrm{~h}$ after the cMORF injection. The clearance effect for each avidin injection was estimated by comparing the group with that avidin injection as the last avidin injection to another group without it (group 1 vs. 2 for the first avidin injection, group 2 vs. 3 for the second, and group 3 vs. 4 for the third).

\section{RESULTS \\ CLEARANCE EFFICIENCY BY AVIDIN WITH VARYING PRETARGETING INTERVAL}

Both prolonging the pretargeting interval and the use of avidin reduced the antibody levels in blood as shown in Figure 1 and normal tissues (not presented), but the use of avidin is more effective. Two-Way ANOVA indicates that the cMORF blood level is significantly decreased both by pretargeting interval prolongation $(p=0.007)$ and avidin administration $(p<0.00005)$. With regard to avidin administration, the result of ANOVA is in agreement with the visual judgment based on the large difference relative to the SD at each interval. The clearance efficiency using avidin as a clearing agent is calculated to be $55-58 \%$, with $>40 \%$ antibody remaining in the circulation. As will be shown, this incomplete clearance is neither due to ineffective binding of avidin nor to the dissociation of the avidin/biotin complex but mainly results from the rapid clearance of avidin from blood to liver.

The cleared and the remaining labeled molecules in the circulation seem to share the same pharmacokinetics. The varying pretargeting interval did not change the clearance efficiency (Figure 1). No significant difference is evident among the clearance efficiencies at the 1-3 day pretargeting intervals.

\section{AVIDIN CLEARABILITY OF THE BIOTIN-ANTIBODY-MORF}

As shown in Figure 2A, the blood concentration of the radiolabeled biotin-CC49-MORF not treated with avidin is much higher than that for the avidin-treated. The percentages cleared from 
circulation (1-the ratio of blood levels with/without avidin) are $65 \pm 3 \%, 84 \pm 1 \%, 89 \pm 1 \%$, and $88 \pm 1 \%$ for $2,30 \mathrm{~min}, 1.5$, and $2 \mathrm{~h}$, respectively. At $0.5 \mathrm{~h}$, the clearance of the avidin-treated antibody is almost complete, although additional slight improvement is observed until $1.5 \mathrm{~h}$. Thus, about $88 \%$ of the avidin-treated biotin-CC49-MORF is clearable. The approximate $12 \%$ of avidin that is non-clearable (that cannot be cleared by avidin) is likely due to the lack of a biotin group.

The high liver accumulation in Figure 2B confirms the previous report that avidin clears the antibody into this organ (Ashwell

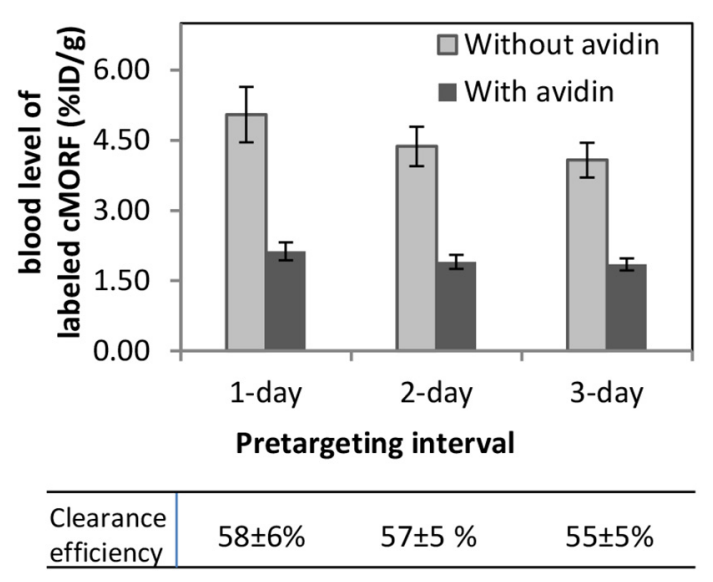

FIGURE 1 | Clearance efficiency at different pretargeting intervals in a pretargeting procedure using biotin-CC49-MORF, avidin, and

$\mathbf{9 9} \mathbf{m}$ Tc-cMORF. The cMORF level is proportional to and used as a measure of the antibody level. The clearance efficiency is defined as the ratio of blood level with/without avidin. and Morell, 1974; Ong et al., 1991). Subsequent clearance out of this organ is slow, as shown in Figure $2 \mathrm{C}$ by the almost identical whole body retention. The $\sim 30 \%$ loss of the radioactivity in the whole body is likely due to the lower affinity of some sequence impurities and therefore the dissociation of the MORF/cMORF complex. Figure 2D demonstrates higher kidney accumulations after $30 \mathrm{~min}$ for the non-avidin-treated antibody as compared to the avidin-treated, likely due to the higher blood concentrations of the antibody shown in Figure 2A.

\section{MULTIPLE INJECTIONS OF AVIDIN}

As shown in Figure 3, additional injections of avidin subsequent to the first further reduce the blood antibody concentration. This indicates that the majority of antibody molecules that survived the first avidin injection are clearable. The collective clearance efficiency following 3 avidin injections is $80 \pm$ $2 \%\left(0.61 / 3.10^{*} 100 \%\right)$. The separate clearance efficiency following each injection is $43 \pm 12 \%(1-1.78 / 3.10), 44 \pm 11 \%(1-$ $0.99 / 1.78)$, and $38 \pm 7 \%(1-0.61 / 0.99)$, respectively. The real clearance efficiency may actually be higher because of the nonclearable portion. A calculation based on a constant clearance efficiency of 51\% (higher than 38-44\%), and the non-clearable portion of $12 \%$ provides the blood concentrations of $3.10,1.71$, $1.03,0.69 \% \mathrm{ID} / \mathrm{g}$. These numbers are in a good agreement to the measured $3.10 \pm 0.24,1.78 \pm 0.33,0.99 \pm 0.07,0.61 \pm$ $0.05 \% \mathrm{ID} / \mathrm{g}$.

\section{DISCUSSION}

In the current investigation, we have demonstrated that biotinylated antibody can be cleared from the circulation completely and rapidly if bound to avidin. To the best of our knowledge, until this investigation it remained a hypothesis,
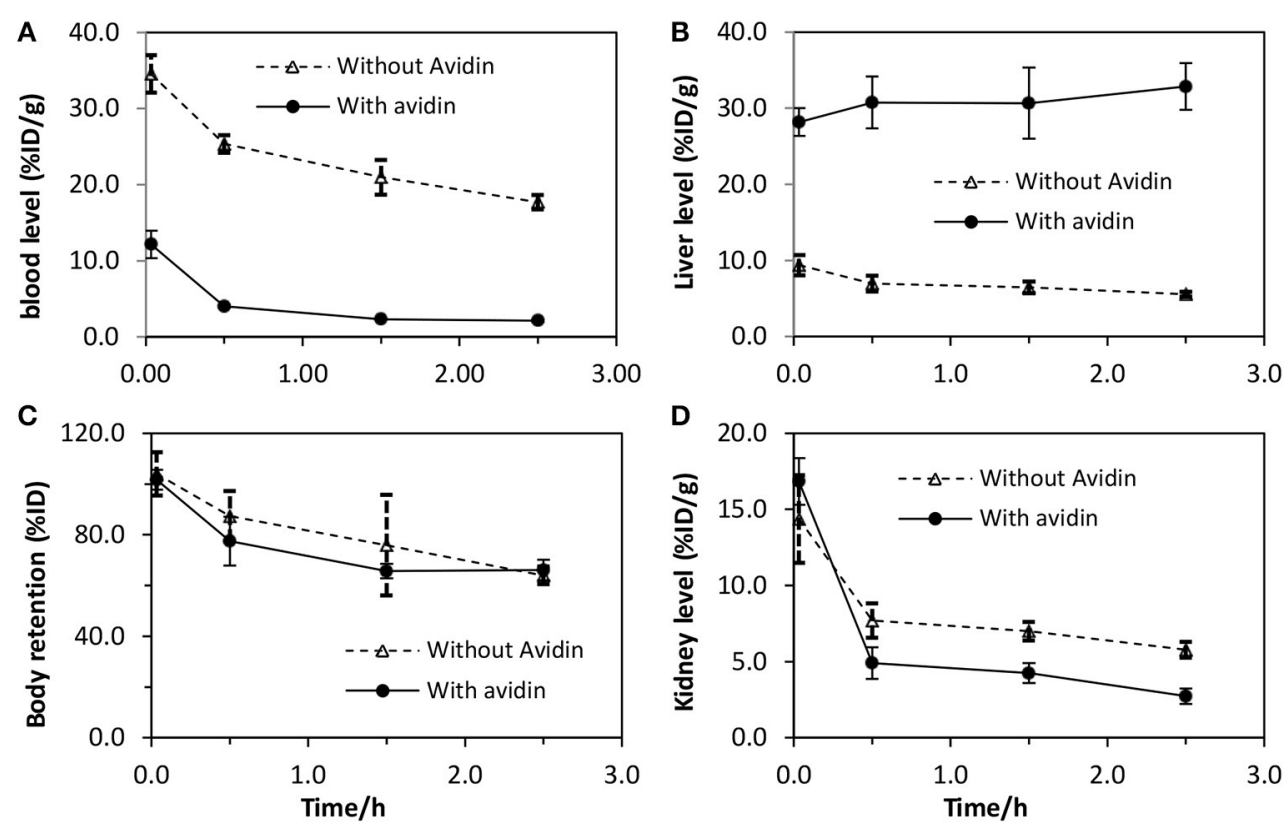

FIGURE 2 | Pharmacokinetics of both avidin-bound and radiolabeled biotin-CC49-MORF vs. radiolabeled biotin-CC49-MORF (no avidin). Shown are pharmacokinetics for (A) Blood, (B) Liver, (C)

whole body retention, and (D) kidney. It mimics how a biotinylated antibody molecule behaves once bound to avidin in blood. 


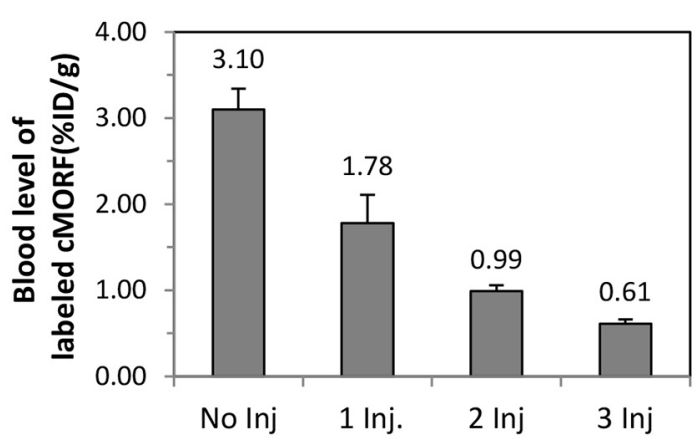

FIGURE 3 | The blood radioactivity levels after the injection of the biotin-Ab-MORF, different numbers of injections of avidin, and the injection of the radiolabeled cMORF. The time of euthanization was at 4 days after initial injection of the pretargeting antibody and $3 \mathrm{~h}$ after injection of radiolabeled cMORF (see Materials and Methods)

although the incomplete clearance of antibody by avidin was known. Yao et al. hypothesized that one of the possible reasons underlying the incomplete clearance might be a too low biotinylation of the antibody (Yao et al., 1995), because avidin would not bind an antibody without biotin. In the current investigation, this possibility was confirmed to be limited to only $\sim 12 \%$ for the MORF-Ab carrying an average of 1.68 biotins per molecule. Another possible explanation Yao et al. proposed was the inaccessibility of the antibody that diffused into the interstitial fluid (extravascular space). As some clearing agents reached a clearance effect of $>90 \%$ (Sharkey et al., 1997; Axworthy et al., 2000), an auxiliary explanation may be the rapid clearance of avidin relative to the diffusion into the extravascular space (Mirallie et al., 2005). It has been reported that most labeled avidin is eliminated from blood within $2 \mathrm{~min}$ in rabbits (Rosebrough, 1993). When the avidin is essentially cleared from circulation, the extravascular antibodies would return. A rebound of the radiolabeled antibody concentration in the blood has been previously observed (Kobayashi et al., 1995).

The hypothesis that temporary inaccessibility of the antibody in the extravascular space, coupled with the rapid clearance of avidin from the circulation, also explains the avidin doseclearance curves (Rosebrough, 1993; Liu et al., 2010). An initial narrow dose range with efficient improvement in antibody clearance may correspond to the range of saturating the biotinylated antibody within the blood pool. The subsequent less effective larger range may reflect the difficulty for the avidin to diffuse across the capillary wall into the extravascular space due to its rapid clearance from the blood.

We have employed a pretargeting system to measure the concentration of the antibody in the current investigation as well as previous studies (Liu et al., 2010). The conventional directlabeling approach (to compare labeled antibody level in normal tissues with and without an injection of clearing agent) would underestimate the clearance efficiency, because radiolabels, particularly radiometals, may have dissociated from antibody and localized within the cells. In this study, we have defined the clearance efficiency. It can also be calculated based on the measurement by pretargeting.
The immediate utility of the findings in this investigation is that it paves the way for using avidin as the clearing agent to develop an improved pretargeting technology. The tri-functional antibody allows for independent binding to the target, to the clearing agent, and to the labeled effector. In some other pretargeting technologies with galactosylated clearing agent, the effector and the clearing agent compete for the same binding site. Although the clearance efficiency by a single injection of avidin is lower than that provided by a single injection of secondary antibody ( $50-60 \%$ vs. $>90 \%$ ), it would not affect the endogenous IgG antibodies in animal and human (Sinitsyn et al., 1989). As the lower clearance efficiency (50-60\%) stems from the too rapid clearance of avidin, a continuous infusion within 1 or $2 \mathrm{~h}$ would achieve a clearance effect suitable for clinical applications.

More importantly, the topic of antibody-trafficking among different compartments and its clearance from circulation are of interest for immunotherapy or targeted chemotherapy ingeneral. The clearance approach may apply similarly to the antibody-drug conjugates for tumor targeting. Clearance of the circulating antibody would greatly reduce the toxicity to normal tissues and therefore may permit higher dose for increased toxicity to the tumor.

\section{CONCLUSION}

Understanding the in vivo interaction between antibodies and their clearing agent should be useful for antibody-based drug discovery. We demonstrated the rapid clearance from blood of every avidin-bound antibody molecule, differentiated the temporary non-clearable and the real non-clearable antibody molecules, and validated the previous hypotheses in the literature. We also quantitated the clearance processes by defining clearance efficiency and using pretargeting as a research tool. Based on the multiple injection study, we suggest a continuous administration (infusion) of the clearing agent as a solution for the temporary non-clearable antibodies.

\section{ACKNOWLEDGMENTS}

This work was partially supported by DK94199 (Alvin C. Powers and Guozheng Liu), DK82894 (Guozheng Liu), CA94994 (Guozheng Liu) DK89527 (Alvin C. Powers), and DK72473 (Alvin C. Powers) from the National Institutes of Health as well as by the grants from the Department of Veterans Affairs (Merit Review to Alvin C. Powers), JDRF, and the Vanderbilt Diabetes Research and Training Center (DK20593). We thank the staff of the Division of Nuclear Medicine, Department of Radiology, University of Massachusetts Medical School for their help with the ${ }^{99 \mathrm{~m}} \mathrm{Tc}$.

\section{REFERENCES}

Ashwell, G., and Morell, A. G. (1974). The role of surface carbohydrates in the hepatic recognition and transport of circulating glycoproteins. Adv. Enzymol. 41, 99-128.

Axworthy, D. B., Reno, J. M., Hylarides, M. D., Mallett, R. W., Theodore, L. J., Gustavson, L. M., et al. (2000). Cure of human carcinoma xenografts by a single dose of pretargeted yttrium-90 with negligible toxicity. Proc. Natl. Acad. Sci. U.S.A. 97, 1802-1807. doi: 10.1073/pnas.97.4.1802

Deng, C., Pan, B., and O'Connor, O. A. (2013). Brentuximab vedotin. Clin. Cancer Res. 19, 22-27. doi: 10.1158/1078-0432.CCR-12-0290 
Goodwin, D. A., Meares, C. F., McCall, M. J., McTigue, M., and Chaovapong, W. (1988). Pre-targeted immunoscintigraphy of murine tumors with indium-111labeled bifunctional haptens. J. Nucl. Med. 29, 226-234.

Goodwin, D. A., Meares, C. F., Watanabe, N., McTigue, M., Chaovapong, W., Ransone, C. M., et al. (1994). Pharmacokinetics of pretargeted monoclonal antibody 2D12.5 and ${ }^{88}$ Y-Janus-2-(p-nitrobenzyl)-1,4,7,10tetraazacyclododecanetetraacetic acid (DOTA) in BALB/c mice with KHJJ mouse adenocarcinoma: a model for ${ }^{90} \mathrm{Y}$ radioimmunotherapy. Cancer Res. 54, 5937-5946.

Karacay, H., Sharkey, R. M., Govindan, S. V., McBride, W. J., Goldenberg, D. M., Hansen, H. J., et al. (1997). Development of a streptavidin-anticarcinoembryonic antigen antibody, radiolabeled biotin pretargeting method for radioimmunotherapy of colorectal cancer. Reagent development. Bioconjug. Chem. 8, 585-594. doi: 10.1021/bc970102n

Kobayashi, H., Sakahara, H., Endo, K., Yao, Z., Toyama, S., and Konishi, J. (1995). Repeating the avidin chase markedly improved the biodistribution of radiolabeled biotinylated antibodies and promoted the excretion of additional background radioactivity. Eur. J. Cancer 31, 1689-9166. doi: 10.1016/09598049(95)00244-D

Liu, G., Dou, S., Akalin, A., Rusckowski, M., Streeter, P. R., Shultz, L. D., et al. (2012). Pretargeting vs. direct targeting of human betalox 5 islet cells subcutaneously implanted in mice using an anti-human islet cell antibody. Nucl. Med. Biol. 39, 645-651. doi: 10.1016/j.nucmedbio.2011.12.001

Liu, G., Dou, S., Chen, X., Chen, L., Liu, X., Rusckowski, M., et al. (2010). Adding a clearing agent to pretargeting does not lower the tumor accumulation of the effector as predicted. Cancer Biother. Radiopharm. 25, 757-762. doi: $10.1089 /$ cbr. 2010.0800

Liu, G., Dou, S., Cheng, D., Leif, J., Rusckowski, M., Streeter, P. R., et al. (2011). Human islet cell MORF/cMORF pretargeting in a xenogeneic murine transplant model. Mol. Pharm. 8, 767-773. doi: 10.1021/mp100382m

Liu, G., Dou, S., He, J., Yin, D., Gupta, S., Zhang, S., et al. (2006). Radiolabeling of $\mathrm{MAG}_{3}$-morpholino oligomers with ${ }^{188} \mathrm{Re}$ at high labeling efficiency and specific radioactivity for tumor pretargeting. Appl. Radiat. Isot. 64, 971-978. doi: 10.1016/j.apradiso.2006.04.005

Mirallie, E., Sai-Maurel, C., Faivre-Chauvet, A., Regenet, N., Chang, C. H., Goldenberg, D. M., et al. (2005). Improved pretargeted delivery of radiolabelled hapten to human tumour xenograft in mice by avidin chase of circulating bispecific antibody. Eur. J. Nucl. Med. Mol. Imaging 32, 901-909. doi: 10.1007/s00259-005-1811-2

Ong, G. L., Ettenson, D., Sharkey, R. M., Marks, A., Baumal, R., Goldenberg, D. M., et al. (1991). Galactose-conjugated antibodies in cancer therapy: properties and principles of action. Cancer Res. 51, 1619-1626.
Rosebrough, S. F. (1993). Pharmacokinetics and biodistribution of radiolabeled avidin, streptavidin and biotin. Nucl. Med. Biol. 20, 663-668. doi: 10.1016/09698051(93)90037-U

Senter, P. D., and Sievers, E. L. (2012). The discovery and development of brentuximab vedotin for use in relapsed Hodgkin lymphoma and systemic anaplastic large cell lymphoma. Nat. Biotechnol. 30, 631-637. doi: 10.1038/ nbt.2289

Sharkey, R. M., Karacay, H., Griffiths, G. L., Behr, T. M., Blumenthal, R. D., Mattes, M. J., et al. (1997). Development of a streptavidinanti-carcinoembryonic antigen antibody, radiolabeled biotin pretargeting method for radioimmunotherapy of colorectal cancer. Studies in a human colon cancer xenograft model. Bioconjug. Chem. 8, 595. doi: 10.1021/ bc970101v

Sinitsyn, V. V., Mamontova, A. G., Checkneva, Y. Y., Shnyra, A. A., and Domogatsky, S. P. (1989). Rapid blood clearance of biotinylated IgG after infusion of avidin. J. Nucl. Med. 30, 66-69.

Wang, Y., Chang, F., Zhang, Y., Liu, N., Liu, G., Gupta, S., et al. (2001). Pretargeting with amplification using polymeric peptide nucleic acid. Bioconjug. Chem. 12, 807-816. doi: 10.1021/bc010 0307

Yao, Z., Zhang, M., Kobayashi, H., Sakahara, H., Nakada, H., Yamashina, I., et al. (1995). Improved targeting of radiolabeled streptavidin in tumors pretargeted with biotinylated monoclonal antibodies through an avidin chase. J. Nucl. Med. $36,837-841$.

Conflict of Interest Statement: The authors declare that the research was conducted in the absence of any commercial or financial relationships that could be construed as a potential conflict of interest.

Received: 23 May 2014; accepted: 02 July 2014; published online: 24 July 2014.

Citation: Dou S, Virostko J, Rusckowski M, Greiner DL, Powers AC and Liu G (2014)

Differentiation between temporary and real non-clearability of biotinylated IgG antibody by avidin in mice. Front. Pharmacol. 5:172. doi: 10.3389/fphar.2014.00172 This article was submitted to Experimental Pharmacology and Drug Discovery, a section of the journal Frontiers in Pharmacology.

Copyright (C) 2014 Dou, Virostko, Rusckowski, Greiner, Powers and Liu. This is an open-access article distributed under the terms of the Creative Commons Attribution License (CC BY). The use, distribution or reproduction in other forums is permitted, provided the original author(s) or licensor are credited and that the original publication in this journal is cited, in accordance with accepted academic practice. No use, distribution or reproduction is permitted which does not comply with these terms. 